\title{
An Oil-water Layer Recognition System Based on Composition Intelligence Computation
}

\author{
Huanglin Zeng ${ }^{1}$, Juan $\mathrm{Li}^{2}$ \\ ${ }^{1}$ Sichuan University of Science and Engineering, 643000, P. R. China \\ ${ }^{2}$ Sichuan University of Science and Engineering, 643000, P. R. China
}

\begin{abstract}
In this paper, a composition intelligence computing method is suggested for an oil-water layer recognition system. The redundant condition attributes are reduced based on rough set attribute simplification algorithm an oil-water layer neural network recognition system can be simplified in order to improve network training speed. A local minimum problem of optimization computation of neural network is improved by a composition GA BP learning algorithm. Simulation result shows that the effect in oil-water layer recognition is improved by the composition intelligence computing method proposed here.
\end{abstract}

Keywords: Attribute reduction, Neural network, Genetic algorithm, Oil-water layer recognition

\section{Introduction}

Pattern recognition of oil-water layer is an important discipline field in oil log interpretation. In the oilwater layer recognition, a lot of well log attributes such as sound wavelength, number of neutron, layer resistance, porosity, oil saturation, permeability may be included in a lot of feature such that they will cause repetition and waste of information. The excessive data will give rise to take up a great deal of storage space and processing time of computer machine. In this case, we must get rid of redundant attribute in order to get the useful information to carry on the recognition [1].

Neural network [7] is widely used in the oilwater layer recognition in which massively parallel processing and nonlinear mapping can self-adaptively clustered for data. The disadvantage of oil-water layer neural network recognition can not get rid of redundant attribute. Therefore, an oil-water layer recognition system calls for a composition intelligence computing method.

In this paper, a composition intelligence computing method is suggested for an oil-water layer recognition system. The concrete innovation work is as follows:

(1) Get rid of redundant condition attribute based on rough set attribute simplification algorithm so that an oil-water layer neural network recognition system can be simplified in order to improve network training speed.

(2) Making use of general algorithm to train the weight-threshold of a back propagation neural network so that a local minimum problem of optimization computation is improved by a composition GA BP learning algorithm.

(3) A composition intelligence computing method can reduce input information space dimensions, decrease operation and simplify topology structure of an oil-water layer recognition system. Simulation result shows that the effect of oil log interpretation of oil-water layer recognition is greatly improved.

\section{An oil-water layer recognition system based on composition intelligence computation}

\subsection{Attribute reduction of sample information}

An information system of an oil log interpretation of oil-water layer recognition is defined as $(U, Q)$, where $U$ is an universe of discourse and $Q$ is a finite attribute set, and $C$ is a condition attribute set such as sound wavelength $\left(C_{1}\right)$, number of neutron $\left(C_{2}\right)$, layer resistance $\left(C_{3}\right)$, porosity $\left(C_{4}\right)$, oil saturation $\left(C_{5}\right)$, permeability ( $\left.c_{6}\right), C=\left\{c_{1}, c_{2}, c_{3}, c_{4}, c_{5}, c_{6}\right\}, D$ is called decision attribute, $D=\left\{d_{i}=i, i=0,1,2,3\right\}$, $0,1,2,3$ respectively denotes soil layer, water layer, oilwater layer and oil layer.

The reduction step of condition attribute set based on rough set [2-5] is as follows:

(1) Dispersing of successive attributes and decision attributes generalization

Decision attribute is known in the oil-water table, namely soil layer, water layer, oil-water layer and oil layer. Changing this decision attribute into successively non-negative integer value. Base on selection complete sample information, this paper separately carries on the corresponding interval partition to each condition attribute according to 
decision attribute type, and gets cut-point by median and cluster point principle, then realizes dispersing of successive attribute.

Attribute sets normalized as follows:

$$
x^{\prime}=\frac{x-x_{\min }}{x_{\max }-x_{\min }}
$$

Where $x$ is actual value of attribute in the table 1 , and $x \in\left[x_{\min }, x_{\max }\right], x_{\min }$ is minimum, $x_{\max }$ is maximum.

(2)Seek core of sample information core $(C)$

Computation equivalence relations ind $(C)$ as follows:

$$
\text { ind }\left(C-\left\{c_{i}\right\}\right)(i=1,2, \ldots)
$$

It gets redundant attributes, then obtains:

$$
\operatorname{core}(C)=\bigcap \operatorname{red}(C)
$$

(3) Seek relative least reduction set

A beginning carries on extending from $\operatorname{core}(C)$, and gradually increases redundant attribute $C_{i}$ in the core, and obtains $R$, if

$$
\operatorname{ind}(R)=\operatorname{ind}(C)
$$

Then $R$ is relative least reduction set.

\subsection{Algorithm of GA train power- threshold of neural network}

An algorithm of GA training power-threshold of neural network $[6,7]$ is realized as follows:

1) Initialization: stochastically produces an initial population $\mathrm{P}=\left\{x_{1}, x_{2}, \cdots, x_{n}\right\} \quad, \quad \forall \quad x_{i} \in P \quad$ is corresponding a group of neural network link power and threshold value. Neuron is coded by bottom-up and left-right (including input layer neuron). Where, $x_{i}$ is initialized by small random number of normal distribution.

2) Evaluation: according to stochastic power and threshold vector corresponding neural network, the global error is defined as:

$$
f=\sum_{p=1}^{k} \sum_{i=1}^{n}\left(d_{p i}-o_{p i}\right)^{2}
$$

Probability of choice chromosome is determined as :

$$
\operatorname{eval}\left(v_{i}\right)=a(1-a)^{i-1} \quad, \quad a \in(0,1) \quad,
$$
$i=1,2, \cdots$, pop_size . $\mathrm{i}=1$ means that chromosome is the best, otherwise i=pop_size. $a=0.05$.

3) Choice operation: base on the rule of wheel gambling, choice operation chooses a chromosome for the new group in every turning gambling.

a. To each chromosome $v_{i}$, calculates its cumulative probability $q_{i}$ :

$$
\left\{\begin{array}{c}
q_{0}=0 \\
q_{i}=\sum_{j=1}^{i} \operatorname{eval}\left(v_{j}\right), i=1,2, \cdots, \text { pop_size; }
\end{array}\right.
$$

b. From the $\operatorname{span}\left(0, q_{\text {pop_size }}\right)$, produces a uniform distribution random number.

c. If $q_{i-1}<r \leq q_{i}$, then chooses $i$ th chromosome $v_{i}\left(1 \leq i \leq p o p \_s i z e\right)$.

d. Repeat b and c operation pop_size times, may obtains pop_size numbers duplication chromosomes. If in pop_size-1 th choice process, has not appeared the first best chromosome, then in the pop_size th, direct selection first best chromosome.

4) Crossover operation: to determine the father of crossover operation, repeats the follow operation from $\mathrm{i}=1$ to pop_size. Produces a uniform distribution random number $\mathrm{r}$ from span [0,1], if $r<p_{c}$, then chooses $v_{i}$ take a father generation. If has chose father generation with $v_{1}^{\prime}, v_{2}^{\prime}, v_{3}^{\prime}, \cdots$ expression, and divides them into $\left(v_{1}^{\prime}, v_{2}^{\prime}\right),\left(v_{3}^{\prime}, v_{4}^{\prime}\right), \cdots$. Crossover form is taken as $x=c \times v_{1}^{\prime}+(2-c) \times v_{2}^{\prime}, y=(2-c) \times v_{1}^{\prime}+c \times v_{2}^{\prime}$, where $x$ and $y$ is descendant.

5) Mutation operation: its process is similar to father generation of choice in the overlapping from $\mathrm{i}=1$ to $\mathrm{pop}_{\text {_ size }}$.

6) Termination condition: if satisfies the termination condition, then stops, and output evolution result. Otherwise return to 3) step.

\subsection{Recognition of oil-water layer based on composition intelligence computation}

The process of recognition of oil-water layer on composition intelligence computation is shows as follows:

1) Sample information preprocessing. Selects the complete sample information, and carries on standardization and normalization based on (2.1)

2) Sample information attributes reduction. Condition attributes are reduced by (2.2)-(2.4).

3) Sets up a neural network of oil-water layer recognition in which the number of neuron of input layer is determined on attribute reduction by rough sets.

4) Genetic algorithm improve nerve network in which the genetic algorithm optimize power-threshold of nerve network. 
5) An oil log interpretation of oil-water layer recognition based on the output result of neural network of oil-water layer recognition.

\section{Simulation and recognition}

In order to analyze the effect of the composition intelligence study method used in oil-water layer recognition, simulation is carried on for oilsk81 well of some oil field [8]. We may know that condition attribute set size is 6 in the oil-water layer recognition, namely sound wavelength $\left(c_{1}\right)$, number of neutron $\left(c_{2}\right)$, layer resistance $\left(c_{3}\right)$, porosity $\left(c_{4}\right)$, oil saturation $\left(c_{5}\right)$, permeability $\left(c_{6}\right), \quad C=\left\{c_{1}, c_{2}, c_{3}, c_{4}, c_{5}, c_{6}\right\}, D$ is called decision attribute, $D=\left\{d_{i}=i, i=0,1,2,3\right\}$, $0,1,2,3$ respectively denotes soil layer, water layer, oilwater layer and oil layer.

According to four types of $\mathrm{D}$, divide $C_{1}$ into four spans [195, 210] , [225, 230] , [201, 240] and [210, 225] , the center of clustering in span are $(0,200]$, $(220,240],(200,210],(210,220]$ by way of clustering in every span. The same argument is encoded into $0,3,1,2$. The other condition attribute is normalized as above.

Base on rough sets, we obtain three reduced sets, namely $\left\{c_{1}, c_{2}, c_{3}, c_{4}\right\} \quad, \quad\left\{c_{1}, c_{2}, c_{3}, c_{5}\right\} \quad$ and $\left\{c_{1}, c_{2}, c_{3}, c_{6}\right\}$.The attribute set $\left\{c_{1}, c_{2}, c_{3}, c_{4}\right\}$ is chosen.

Sets up a three layer 4-15-4 neural network in which the network of oil-water layer recognition in which the number of neuron of input layer is determined on $\left\{c_{1}, c_{2}, c_{3}, c_{4}\right\}$. Output layer of forward neural network has four points, thus $(1,0,0,0),(0$, $1,0,0),(0,0,1,0),(0,0,0,1)$ respectively denotes the desired outputs of soil layer, water layer, oil-water layer and oil layer.

An initial population and power-threshold of nerve network of oil-water layer recognition is optimized genetic algorithm. The recognition error gets the expecting precision when the training algebra achieved $100^{\text {th }}$ generation. The recognition error of oil-water layer recognition system is shown as Fig.1. An oil log interpretation of oil-water layer recognition based on the output result of neural network is shown as table 1.

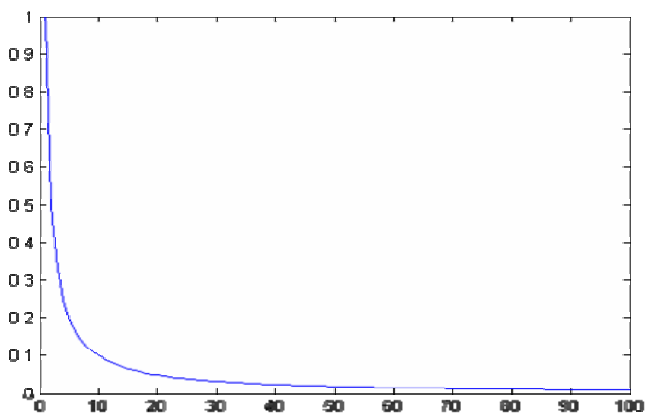

Fig.1 recognition error.

Base on table 1, recognition rate of composition intelligence computing method reaches $92.86 \%$, but recognition rate of traditional BP neural network can reach 71.43\%, obviously, application effect of composition intelligence computing method is better than the traditional BP neural network.

\section{Summaries}

Base on attribute reduction of rough set, the composition intelligence computing method eliminates the redundant attributes and carries on training power and threshold by genetic algorithm in the oil-water layer recognition, which avoids the disadvantage that random select the initial condition and weightthreshold of back propagation (BP) neural networks will result of a local minimum problem of optimization computation. Result of simulation shows that the composition intelligence computing method is practical and feasible, effect is remarkable in the oilwater layer recognition. Furthermore, composition algorithm has the advantages of cost saving and enhancement processing speed.

\begin{tabular}{ccccccccccccccc}
\hline NO & 1 & 2 & 3 & 4 & 5 & 6 & 7 & 8 & 9 & 10 & 11 & 12 & 13 & 14 \\
\hline $\begin{array}{c}\text { Actual- } \\
\text { result }\end{array}$ & 0 & 0 & 0 & 0 & 0 & 0 & 1 & 1 & 2 & 2 & 2 & 2 & 3 & 3 \\
$\begin{array}{c}\text { traditional- } \\
\text { BP }\end{array}$ & 0 & 0 & 0 & 2 & 0 & 0 & 3 & 1 & 1 & 2 & 0 & 2 & 3 & 3 \\
$\begin{array}{c}\text { Composition } \\
\text { intelligence }\end{array}$ & 0 & 0 & 0 & 0 & 0 & 0 & 1 & 1 & 1 & 2 & 2 & 2 & 3 & 3 \\
\hline
\end{tabular}

Table1: Result of recognition for oilsk85 well. 


\section{Acknowledgement}

This work is partially supported by Foundation of Fundamental Science Application of Sichuan Province in China (Grant No. 07JY029-027).

\section{References}

[1] H.L Zeng, R. Swiniarski, A neuro-fuzzy classifier based on rough sets, Intelligent Information Processing Web Mining Proceedings, IIPWM, Poland, pp. 541-549, 2005.

[2] H.L Zeng, H.Y. Lan, X.H. Zeng, Redundant data processing based on rough-fuzzy approach, Rough Sets and Knowledge Technology, Springer-Verlag Berlin Heidelberg, Germany, pp.156-161, 2006.

[3] H.L. Zeng, Z.R. Yuan, X.H. Zeng, A new method of selection and reduction of system feature in pattern recognition based on rough sets, Journal of Communication and Computer, Aug., 3(8):25-28, 2006.

[4] K. Cios, W. Pedrycz, R. Swiniarski, Data mining methods for knowledge discovery, Kluwer academic publishers, 1998.

[5] W. Skarbek, Rough sets for enhancements of local subspace classifier, Inter. Journal, Neurocomputing, 36:67-84, 2001

[6] R. Jensen, Q. Shen, Fuzzy2rough sets for descriptive dimensionality reduction, Proc of the 11th Int Conf on Fuzzy Systems. HI: Honolulu, pp.29-34, 2002.

[7] Martin T. Hagan, Howard B. Demuth, Mark Beale. Neural Network Design. Citic Publishing House, 2002.

[8] H.X. Guo, K.J. Zhu, Extracting fuzzy rules of oil-bearing formation based on soft computing, Proc of CRSSC-CWI-CGrC'2007, 2007. (in Chinese) 